\title{
A method of judging the sustainability of a country based on SVM
}

\author{
Guojin Zhao \\ School of North China Electric Power University, Baoding 071000, China \\ 15830939181@163.com
}

Keywords: SVM; hyper plane.

\begin{abstract}
This paper explains and demonstrates how to distinguish between sustainable countries and unsustainable ones by using sport vector machine (SVM). There are many methods to analyze and judge the sustainability at home and abroad. However, considering that there only exists methods to judge the sustainability of a country according to one indicator, I come up with a quantitative and comprehensive model to analyze the sustainability of a country. Firstly, I choose nine indicators which include life expectancy at birth, food production index, improved water source, unemployment (of total labor), GDP per capita, poverty headcount ratio, electric power consumption (kwh per capita), $\mathrm{CO} 2$ emissions (metric tons per capita) and forest area (\% of land area). And the indicators I choose cover four aspects: economy, environment, resource and society, which can approximately represent the sustainability of a country. Then use the selected eight countries whose sustainability is clear to get the hyper plane of sport vector machine. Finally, use the method to classify another ten countries. The result of classification is consistent with the actual situation, which shows the accuracy of the model. I use a lot of experimental data to prove the accuracy and rationality of the model. The model in the paper helps us to obtain the sustainability of a country.
\end{abstract}

\section{Introduction}

The concept of sustainable development was introduced by the 1987 Brundtland Report [1] as "development that meets the needs of the present without compromising the ability of future generations to meet their needs." The resources that are available to humanity are finite. Hence, with the increasing of the population and consumption, it becomes more and more important to implement a sustainable development strategy.

Though there exists some methods to analyze sustainability such as ESI, EF EMSI, SEI [2], these methods are only one-sided and cover a few indicators related to the sustainability.Thus, they are not comprehensive and not convincing to a certain extent. There isn't a comprehensive analysis system of the sustainability of a country. And the paper offers an effective method SVM to distinguish between sustainable countries and less sustainable ones.

Support vector machine was proposed by Corinna Cortes and Vapnik in 1995. And it is used to solve the small samples, nonlinear and high dimensional pattern recognition, which shows many unique advantages. It can be applied to the function fitting problems and other machine learning problems.

Collect indicators from different aspects and their corresponding data. I use SVM to classify the sustainability of a country. And programming based on SVM can help us classify between sustainable countries and unsustainable ones. And it is necessary to improve the evaluation theory for sustainable development.

\section{The index set}

We can see that the nine indicators [3] above cover economy, society, environment and resources of a country, which almost include all aspects that can reflect the sustainability of a country. Therefore, the model is comprehensive and reasonable. 


\section{The principle of SVM}

Firstly, choose nine indicators which can reflect the sustainability of a country. Then we use SVM [4] to distinguish unsustainable countries from sustainable ones. SVM is a pattern recognition method based on statistical learning theory, which is widely used in the field of pattern recognition. Its main idea is to find a hyper plane by the known samples that enable it to divide the two sorts of points correctly as many as possible. And at the same time it makes the two divided sorts of points be away from the separating surface to the furthest.

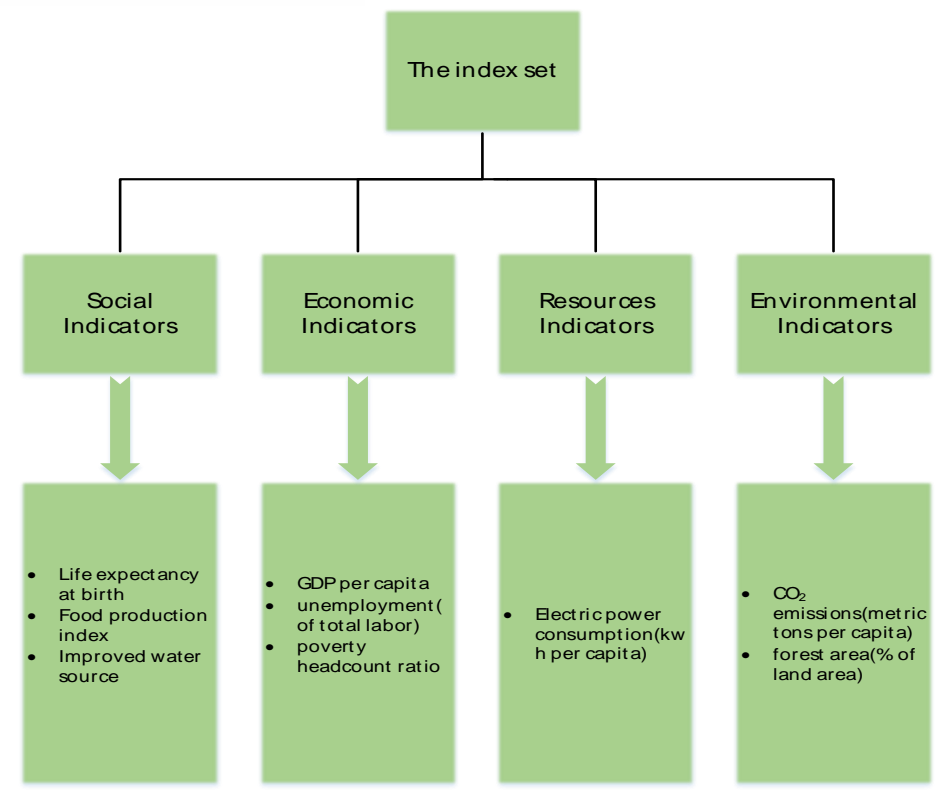

Fig.1 The index set

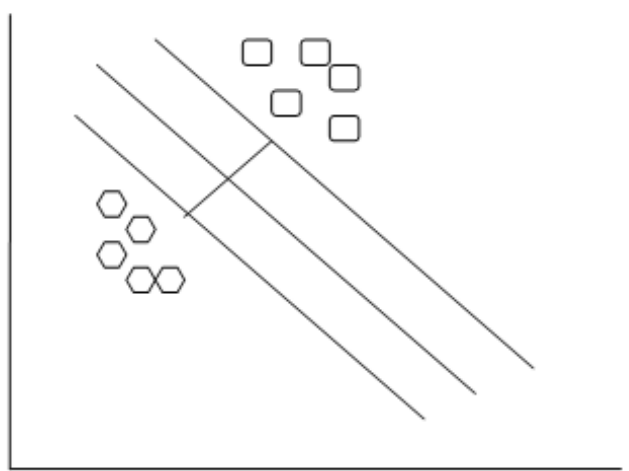

Margin $=2 /\|w\|$.

Fig. 2 The hyper plane

\section{Use selected samples to get the hyper plane}

Choose Australia, Canada, Luxembourg, Switzerland, Ethiopia, Burundi, Malawi and Uganda as the known samples [5].

Table 1 The sustainability of our samples

\begin{tabular}{c|c}
\hline Sustainable & Unsustainable \\
\hline Australia & Ethiopia \\
Canada & Burundi \\
Luxembourg & Malawi \\
Switzerland & Uganda \\
\hline
\end{tabular}

Using the nine indicators above of these samples, we could obtain the appropriate hyper plane of SVM. And the hyper plane can define clearly when a country could be recognized as a sustainable development one. Sign the eight known observation samples as $\left(t_{1}, g_{1}\right),\left(t_{2}, g_{2}\right), \cdots,\left(t_{8}, g_{8}\right)$ and $t_{i}$ is 
composed of nine attributes. If $g_{i}=1$, it means that the sample to be tested is an unsustainable country. If $g_{i}=-1$, it stands for a sustainable one.

It is known that $w^{T} x+b=0, w, x \in R^{9}, b \in R, w, b$ is uncertain, and they satisfy the following relations:

$$
\left\{\begin{array}{l}
w^{T} t_{i}+b \geq 1, g_{i}=1 \\
w^{T} t_{i}+b \leq-1, g_{i}=-1
\end{array}\right.
$$

Switch the equation above simply. Then we can get $g_{i}\left(w t_{i}-b\right) \geq 1$.

To make the two kinds of clarifications be farthest away from the hyper plane, we can get the following equation:

$$
\max \frac{2}{\|w\|} \Rightarrow \min \frac{1}{2}\|w\|^{2}
$$

Then the SVM model is as follows:

$$
\begin{aligned}
& \min \frac{1}{2}\|w\|^{2} \\
& \text { s.t. } g_{i}\left(w^{T} t_{i}+b\right) \geq 1, i=1,2, \cdots, n
\end{aligned}
$$

By obtaining the optimal value, we can get the classification function:

$$
g(x)=\operatorname{sgn}\left(w^{* T} x+b^{*}\right)
$$

The above model is a quadratic programming model. When indicators of these eight countries are brought into $\mathrm{g}(\mathrm{x})$, if the result is -1 , then the country is sustainable, otherwise it is an unsustainable country.

We obtain the most appropriate hyper plane according to our programming, and the hyper plane is equal to the standard which distinguishes clearly the sustainable countries from unsustainable ones.

\section{Model test}

Take all samples back to the classification function of the SVM. And the results are all correct. The rate of error is zero.

Considering that the number of known samples which we used to build SVM is small, we add 10 countries to expand the number of samples. We collect relevant data of America, China, Japan, Nepal, New Zealand, Singapore, India, South Africa, Burma and Russia, and take them into SVM to rebuild the classification function. At present, the eighteen sample countries can stand for different degrees of development around the world. And the model is more ideal compared with the original one we built before.

Table 2 The results of classification

\begin{tabular}{c|c|c|c}
\hline Sustainable & $g(x)$ & Unsustainable & $g(x)$ \\
\hline America & -1 & China & 1 \\
Japan & -1 & Nepal & 1 \\
New Zealand & -1 & India & 1 \\
Singapore & -1 & South Africa & 1 \\
& & Burma & 1 \\
\hline
\end{tabular}

The results above are consistent with the common evaluation internationally on the sustainability of these countries. At the same time, the model distinguishes some countries whose sustainability is vague such as Chinese and Russia clearly. So the model is effective in classification. 


\section{Summary}

The method uses pattern recognition method and it is relevant to intelligent analysis. The difference between this model and the previous methods is that it is comprehensive and covers all aspects that can reflect a country's sustainability based on SVM. The paper offers us a quantitative and comprehensive system to analyze the sustainability of a country, which will undoubtedly have a great significance in modern society. The new method is necessary to improve the evaluation theory for sustainable development. And it helps us to know about the sustainability of a country and helps to build a more sustainable world.

\section{References}

[1]World Commission on Environment and Development (WCED). 1987. Our Common Future. New York: Oxford University Press, 1987, 8.

[2]Hao Cui, Li Hongyuan, Meng Weiqing, Comparison and Analysis on Different Methods of Sustainability Evaluation at Home and Abroad. J. China population, resource and environment, 2012.

[3] http://data.worldbank.org/

[4]Si Shoukui,Sun Xijing(2011).Mathematical Modeling(4thed).National Defense Industry Press. ISBN 978-7-118-07647-9

[5]Wang Haiyan. The latest Index System to Measure Sustainable Development.[J].China Population, Resources and Environment,1996,6(1) 